\title{
Tumour Metabolism: An Emerging Therapeutic Target for
}

\section{Cancer Treatment}

\section{Sonker P and Kumar A* \\ Department of Zoology, Banaras Hindu University, India}

*Corresponding author: Ajay Kumar, Department of Zoology, Institute of Science, Banaras Hindu University, Varanasi-221005, India; Email: ajayzoo@bhu.ac.in; ajaysastri37@gmail.com

\section{Mini Review}

Malignant cells are highly proliferative in nature and display invasion and metastatic properties. Hence, they need rapid energy and huge amount of macromolecules for their development and progression. They fulfil their energy and macromolecular demands either by speeding up/down or switching on /off the various metabolic pathways. Therefore, altered tumor cell metabolism is considered as one of the emerging hallmark features of cancer cells. Most of the cancerous cells usually show dependency on glycolysis, glutaminolysis and lipid biosynthetic pathways.

Several studies indicate an indispensable role of glycolytic pathway in the progression of cancer via supplying rapid ATP, precursors for macromolecular synthesis [1,2]. Moreover, the high glycolytic rate plays a central role in the development of acidified tumor microenvironment (TME) via generating lactate as an end product [3]. Further, the intracellular lactate produced through glycolytic pathway is transported outside with the help of monocarboxylate transporter which leads to acidification of TME. The acidified tumor microenvironment induces immunosuppression, apoptosis of surrounding normal cells and invasion \& metastasis via activating the matrix-metalloproteases $[3,4]$. Due to these benefits, malignant cells perform glycolysis even in the presence of sufficient oxygen, a phenomenon first time observed by Nobel laureate Otto Heinrich Warburg and thus called as 'Warburg's effect' and popularly known as 'aerobic glycolysis'. Cancerous cells maintain a high rate of glycolysis through enhancing the expression of various glycolysis regulatory molecules, namely glucose transporter, hexokinase, phosphofructokinase, pyruvate kinase, lactate dehydrogenase pyruvate dehydrogenase kinase, and monocarboxylate transporter [2]. Recently, several glycolytic regulatory molecules are being targeted either by their inhibitors or by respective siRNAs in cancers of various origins, for the development of safer and effective targeted therapeutic strategies for the treatment of cancer patients.

The role of glutaminolysis has also been observed in various malignancies and most of the cancerous cells are found glutamine addicted and show dependency on glutamine metabolism to fulfil their bioenergetics and biosynthetic requirements $[5,6]$. The reason behind showing their interest in glutamine is due to its easy availability and ability to supply various precursors and intermediate molecules for bioenergetics and macromolecular synthesis pathways. Interestingly, in mammals, the level of glutamine is high in blood and muscles among the all amino acids. Cancer cells utilize glutamine for the production of numerous metabolites, nonessential amino acids, antioxidants for the removal of reactive oxygen species, and nitrogenous bases and fatty acids for the synthesis of nucleic acid and lipids, respectively $[5,6]$. Therefore, glutamine metabolism is emerging as a novel therapeutic target for the treatment of numerous cancers.

Reports also suggest a crucial role of lipid metabolism enzymes in tumorigenesis and tumor progression [7-9]. This could be due to following facts: i) an important component of plasma membrane, ii) one of the key energy source during the nutrient deprivation and iii) crucial regulators of various signaling pathways $[7,8]$. Therefore, due to above discussed benefits; cancer cells modulate 


\section{International Journal of Zoology and Animal Biology}

their lipid metabolic pathways according to their requirement during the course their development and progression. Further, the augmented expression/activity of lipid metabolism enzymes, such as ATP citrate lyase (ACLY), acetyl-CoA carboxylase (ACC), fatty acid synthase (FASN), and carnitine palmitoyltransferase 1 (CPT1) is found in malignancies of various origins $[8,10]$. Thus, cancer-specific lipid metabolism enzymes may be exploited as diagnostic markers or therapeutic targets for developing the diagnostic tools or designing the targeted therapeutic protocols against cancers.

Therefore, reprogrammed tumor cell metabolism plays a crucial role in supporting the unhindered proliferation of cancer cells via accelerating the pathways involved in the generation of biosynthetic materials, rapid energy, and membrane biogenesis. Hence, targeting of tumor metabolism could help in designing the effective and promising therapeutic strategies for the treatment of cancer patients.

\section{Acknowledgement}

We thankfully acknowledge fellowship support to Pratishtha Sonker [Award No. F117.1/201516/RGNF201 517SCUTT4822/[SAIII/Website)] from UGC, New Delhi. Funding from University Grants Commission and Department of Science and Technology, New Delhi, India, in the form of UGC-Start-Up Research (F. No. 30370/2017 (BSR)) and Early Career Research Award (ECR/2016/001117) is highly acknowledged. Financial support from ISLS and UGC-UPE, Banaras Hindu University is also acknowledged. We also acknowledge UGC-CAS and DST-FIST program to the Department of Zoology, Banaras Hindu University, India.

\section{References}

1. Pavlova NN, Thompson CB (2016) The Emerging Hallmarks of Cancer Metabolism. Cell Metab 23(1): 27-47.
2. Kroemer G, Pouyssegur J (2008) Tumor cell metabolism: cancer's Achilles' heel. Cancer Cell 13(6): 472-482.

3. Jiang B (2017) Aerobic glycolysis and high level of lactate in cancer metabolism and microenvironment. Genes Dis 4(1): 25-27.

4. Webb BA, Chimenti M, Jacobson MP, Barber DL (2011) Dysregulated pH: a perfect storm for cancer progression. Nat Rev Cancer 11(9): 671-677.

5. Jin L, Alesi GN, Kang S (2016) Glutaminolysis as a target for cancer therapy. Oncogene 35(28): 36193625 .

6. Yang L, Venneti S, Nagrath D (2017) Glutaminolysis: A Hallmark of Cancer Metabolism. Annu Rev Biomed Eng 19: 163-194.

7. Corbet C, Feron $O$ (2017) Emerging roles of lipid metabolism in cancer progression. Curr Opin Clin Nutr Metab Care 20(4): 254-260.

8. Luo X, Cheng C, Tan Z, Li N, Tang M, et al. (2017) Emerging roles of lipid metabolism in cancer metastasis. Mol Cancer 16(1): 76.

9. Baenke F, Peck B, Miess H, Schulze A (2013) Hooked on fat: the role of lipid synthesis in cancer metabolism and tumour development. Dis Model Mech 6(6): 1353-1363.

10. Beloribi Djefaflia S, Vasseur S, Guillaumond F (2016) Lipid metabolic reprogramming in cancer cells. Oncogenesis 5: e189. 